\title{
Ayenia Mansfeldiana (Herter) Herter ex Cristóbal nov. comb. (Sterculiaceae)
}

\author{
por CARMEN L. CRISTÓBAL'
}

\begin{abstract}
Summary: The new combination Ayenia Mansfeldiana (Herter) Herter ex Cristóbal is proposed based in Tragia Mansfeldiana Herter.
\end{abstract}

Herter en 1937 publicó Tragia Mansfeldiana sobre la base de tres ejemplares coleccionados en Uruguay. La descripción es breve ya que no vio flores, pero la acompañó con dibujos de una pequeña rama, hoja, mericarpio y semilla. Croizat en 1943, excluye a esta especie de las Euforbiáceas y la considera igual a Ayenia pusilla L., especie que según mi criterio está limitada a Ecuador y $\mathrm{N}$ de Perú. Más tarde Herter (1957), publica nuevamente la ilustración $n^{\circ} 6$ de su artículo de 1937, con el nombre de "Ayenia Mansfeldiana Hert.». Si bien este autor no cita el basónimo como lo requiere el código de nomenclatura, considero que la publicación de la misma figura es una clara referencia al nombre anterior, por lo cual es justo considerar a Herter el autor de esta nueva combinación

Ayenia Mansfeldiana (Herter) Herter ex Cristóbal, nov. comb. Herter, Flora ilustrada del Uruguay 13: 568, fig. 2189. 1957.

Tragia Mansfeldiana Herter, Revista Sudamer. Bot. 5(1-2): 34, fig. 6. 1937. Croizat, J. Arnold Arbor. 24: 189. 1943.

Ayenia pusilla L. var. glabra K. Schum. Fl. bras. 12(3): 106. 1886.

Ayenia Ekmanit Cristóbal, Opera Lilloana 4: 145148, fig. 50. 1960.

Material seleccionado. REP. ORIENTAL DEL URUGUAY. Artigas. Catalán. Sicc. soli exp. Camp. sax., 100-200 m s.m., XI.1927, Herter 82725 (G, holotipo). Tacuarembó. Tambores. Sax./arg. Subsicc. ill. 300 m s.m. camp. Il.1937, Herb. Hertei [9] 8255 (G, paratipo). Salto. Arapey. In Sax. ripar. Subsicc. ill. 60 m s.m., IV.1949. Herter 50767 (G). ARGENTINA. Misiones. Depto. Candelaria, Campo San Juan, entre Santa Ana y Candelaria, 26.XI.1958, Cristóbal \& al. 26 (CTES,LIL, tipo de $A$. Ekmanii).

Obs. Ayenia Mansfeldiana, es propia de lugares pedregosos o arenoso-pedregosos. Su área

\footnotetext{
' Instituto de Botánıca del Nordeste (CONICET-UNNE) C.C. 209, 3400 Corrientes Argentina.
}

abarca centro y norte de Paraguay, S de Misiones, Corrientes y Entre Ríos, en Argentina, S de Rio Grande do Sul en Brasil, y Uruguay (Cristóbal, 1960).

Se trata de una especie variable en lo que se refiere al porte, a la forma y pubescencia de las hojas y al tipo de pelos del tallo. El tipo de $A$. Ekmanii y el de A. Mansfeldiana tienen hojas redondeadas y pequeñas. En el primero el epifilo es glabro, y en el segundo puede ser glabro o con pelos simples o de dos brazos. En ambos el tallo tiene pelos adpresos. El paratipo Herb. Herter [9]8255 tiene hojas redondeadas y el epifilo con pelos simples o de dos brazos. En cuanto al paratipo Herter 3085 (G!), es una pequeña rama con dos hojas y sin fiores que pertenece a una Euforbiácea ${ }^{2}$.

En Paraguay, S de Misiones, Corrientes y Entre Ríos, predominan plantas postradas con hojas orbiculares o suborbiculares, pequeñas, siendo el epifilo glabro o con escasos pelos dispersos. Desde aproximadamente el centroeste de Corrientes hacia el S y SE del área, aparecen ejemplares con hojas más grandes y alargadas hacia el extremo de ramas que son erguidas; el epifilo frecuentemente tiene pelos simples o estrellados de pocos brazos, y el tallo puede presentar pelos desordenados y más largos que en las plantas del norte del área. Geográficamente no hay un límite neto, ni las variantes están bien correlacionadas entre sí. Pueden presentarse plantas con hojas grandes, alargadas, con el epifilo subglabro y plantas con todas las hojas redondeadas y con abundantes pelos simples o de varios brazos en el epifilo. En Corrientes, las poblaciones de Tres Cerros son el extremo de la variabilidad, ei tamaño de las hojas es el máximo y el epifilo es pubescente.

2 Agradezco al Dr. A. Costa Aliem y a la Prof. N. Bacigalupo la información que recogieron durante sus trabajos en Euforbiáceas. 